
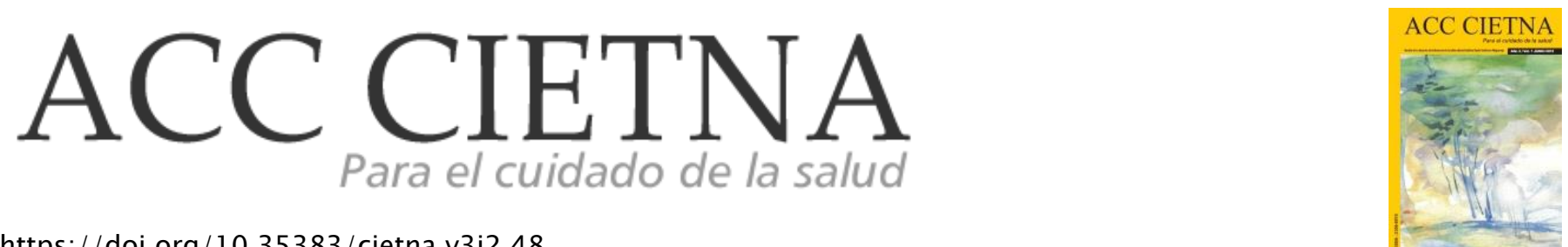

https://doi.org/10.35383/cietna.v3i2.48

\title{
Influencia del programa educativo sobre contaminación por residuos sólidos en el aprendizaje de los estudiantes del sexto grado de una institucion educativa pública
}

\section{Ybañez Nuñez Lizeth Carolina1, Edquén Aquino Diana Carolina² ${ }^{1}$ Carhuajulca Quispe Doris ${ }^{3}$}

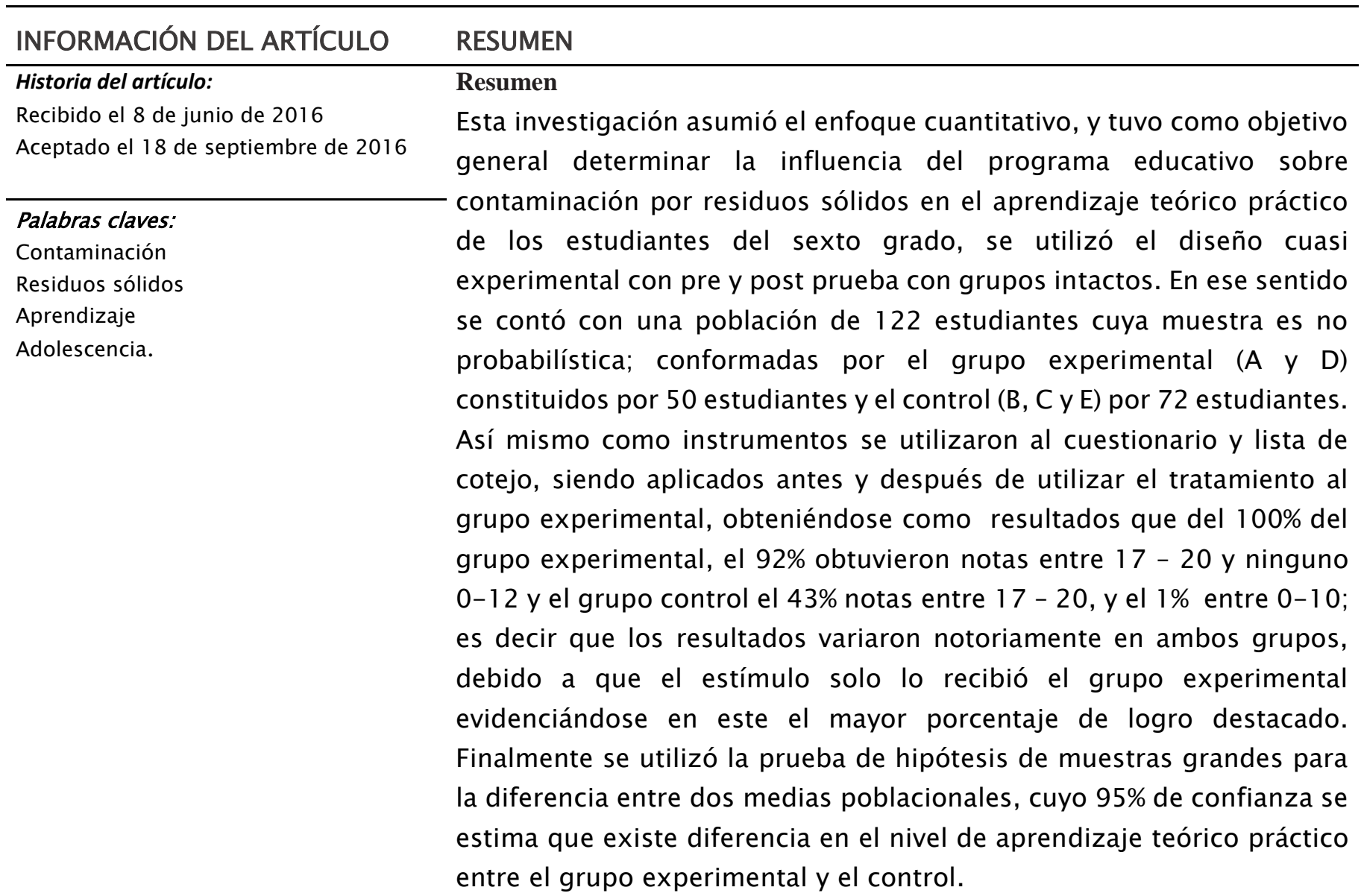

\footnotetext{
${ }^{1}$ Licenciada en Enfermería. Instrumentista Quirúrgico en Cirugía General \& Laparoscopica. Email: caliz_yn@hotmail.com.

${ }^{2}$ Licenciada. Enfermera con trabajo independiente, Chiclayo, Perú. Email: dedquen@gmail.com

${ }^{3}$ Magister en Enfermería. Docente de la Escuela de Enfermería de la Universidad Católica Santo Toribio de Mogrovejo, Chiclayo, Perú. Email: rmoncada@usat.edu.pe
} 
Influence of pollution on education program solid waste in learning student of sixth grade of a public educational institution

\section{ABSTRACT}

Keywords:

Pollution

Solid Waste

Learning
The research titled Influence of educational program on solid waste pollution learning of sixth graders IE Monsignor Juan Tomis Stack, was quantitative, whose overall objective was to determine the influence of the educational program on solid waste pollution in the theoretical and practical learning of sixth graders, the quasi-experimental design was used with pre and post test with intact groups. In that sense it had a population of 122 students whose ample is not probabilistic; formed by the experimental group ( $A$ and $D$ ) consisting of 50 students and control (B, C and E) by 72 students. Also as instruments to the questionnaire and checklist were used, being applied before and after using the treatment experimental group obtained as results that $100 \%$ of the experimental group, $92 \%$ obtained notes from 17 to 20 and none $0-12$ and the control group $43 \%$ notes between 17 to 20 , and $1 \%$ from $0-10$; This means that the results varied markedly in both groups, because only encouragement he received the experimental group evidenced in this the highest percentage of outstanding achievement. Finally hypothesis testing large samples for the difference between two population means was used, whose $95 \%$ confidence is estimated that there is a difference in the level of theoretical and practical learning between the experimental and control group.

\section{Introducción}

En la actualidad la población mundial excede los 5 mil 300 millones de habitantes y se espera que llegue a los 8 mil 500 millones para el año 2025. Durante los próximos 20 años nuestros suelos, bosques $y$ otros recursos naturales experimentarán una presión creciente como resultado de la suma de 3 mil millones de personas que necesitarán alimentos, vivienda, educación y empleo. ${ }^{1}$
Actualmente la humanidad consume un $25 \%$ más de los recursos que la naturaleza produce anualmente. A este ritmo, en el 2050 , el consumo superará en un $50 \%$ la producción de la Tierra', es decir estamos degradando poco a poco los ecosistemas naturales a un ritmo sin precedentes debido al abuso o mal uso de los recursos del planeta lo han puesto en peligro, siendo así que el aire y el agua se agoten, los bosques se estén reduciendo y muchas especies animales se estén extinguiendo por la caza, pesca y la destrucción de sus hábitat naturales; aparte del calentamiento global y cambios climáticos². 
Sin embargo la persona al realizar dichas actividades por satisfacer sus necesidades produce sustancias de desecho que pueden afectar su entorno, convirtiéndose en factores que influyen negativamente en su salud, siendo uno de ellos el desconocimiento en la forma de proteger el medio en que vive o se educa; de allí que existen actividades humanas que desequilibran un entorno saludable para las personas como son los desechos y la contaminación, siendo el principal centro de atención, donde están claramente identificados como factores relacionados con la exposición a toxicidad y por ende riesgo a contraer un tipo de enfermedad, pudiendo de esta manera desencadenar un final fatal para el ser humano. ${ }^{3}$ Esta situación de riesgo para la salud exige mayor importancia de mantener el cuidado del medio ambiente, siendo preocupación a nivel mundial, donde se han identificado los principales problemas con la intervención humana en su mayor porcentaje, aunque en algunas ocasiones se tenga la intencionalidad de mejorar el nivel y calidad de vida de las personas. ${ }^{4}$

Percibiéndose los principales problemas de salud relacionados con la intervención humana, según la OMS, refiere que las enfermedades, son atribuidas a los riesgos ambientales más importantes, y se estimó el número de fallecidos y enfermos por causa de factores como el uso de agua insalubre y un saneamiento deficiente, por el cual cada año mueren más de tres millones de niños por causas $y$ afecciones relacionadas con el medio ambiente 5 .

Así mismo, la constante exposición a factores de riesgo que derivan de la contaminación, para algunos países de Latinoamérica como Brasil, Argentina y Chile, son el inadecuado saneamiento básico que contribuyen a un gran riesgo a la morbi-mortalidad y la discapacidad infantiles asociadas a las enfermedades respiratorias agudas, enfermedades diarreicas, traumatismos físicos, intoxicaciones, enfermedades transmitidas por insectos e infecciones perinatales; siendo Chile unos de los países destacados en cuanto manejo de residuos sólidos, impartiendo programas de un adecuado manejo en colegios como el "Polivalente Elisa Valdés", incentivando y evaluando constantemente, generando cada vez más la toma de conciencia, e incluso tomando medidas más sofisticadas para realizar el compostaje de algunos residuos alimentarios y así disminuir una parte importante de los residuos sólidos de su ambiente. ${ }^{6}$

De la misma manera la contaminación en países como Bolivia, Perú, Ecuador, resaltando este último como el país con más alto porcentaje de contaminación, el problema ya no se denomina un riesgo para la salud, sino como un problema existente; ya que están presenten enfermedades antes mencionadas y como consecuencia corresponde un tercio de las muertes que se deben directamente a causas ambientales, por los cuales los factores En el Perú, en los últimos años, se ha incrementado el deterioro del medio ambiente, específicamente en Lima Metropolitana, donde a través del último censo la población urbana ha crecido y la calidad de vida del poblador se ha visto disminuida, motivado por el incremento de las actividades productivas y de servicios, las cuales crecieron sin tener en cuenta estudios de impacto ambiental ${ }^{8}$, según datos estadísticos existen en mortalidad el $40 \%$ por malaria, $41 \%$ por infecciones respiratorias inferiores y un $94 \%$ por enfermedades diarreicas; teniendo en cuenta que estas tres causas a nivel mundial son denominadas causas de mortalidad en la niñez. ${ }^{9}$

Mientras que esta problemática está presente en Lima, se está propagando cada vez más en otros lugares internos del país, así tenemos al departamento de Lambayeque, que no se encuentra ajeno a esta realidad, donde el medio ambiente también está siendo afectado, específicamente en la provincia de Chiclayo, donde el servicio de limpieza pública es financieramente insostenible produciendo diariamente $277.7 \mathrm{Kg}$. de residuos sólidos en botaderos a cielo abierto; produciendo así contaminación ambiental ${ }_{10}$. De la misma manera este acumulo de residuos de todo tipo que se encuentran en las calles, ríos, colegios, 
etc.; está causando enfermedades, como: Fiebre tifoidea, Disenterías, entre otros. La cual afecta especialmente a los niños entre 5 a 12 años de edad, teniendo un alto porcentaje de muerte por esta causa. 11

Por otra parte analizando la realidad de los colegios, con la edad en la que se encuentra, se afirma que el ser humano desde edades tempranas como, la niñez y adolescencia, adquieren hábitos de higiene tanto negativos como positivos en cuanto al cuidado del medio ambiente, resaltando que la familia es la unidad básica donde se adquieren principalmente estos hábitos, y el colegio donde modifican dichos hábitos adquiridos, con ayuda de la asignatura denominada ciencia tecnología y ambiente, establecido en el plan de estudios del Ministerio de Educación ${ }^{5}$.

Pero a pesar de la educación que reciben en la familia y modificada en el centro de estudios, no lo aplican, debido a la influencia de la sociedad, en relación a los hábitos de la conservación ambiental, y el no saber y no tener conciencia del cuidado que le debemos al medio en el que vivimos, siendo insuficiente el incentivo que reciben éstos por mantener su medio ambiente saludable. ${ }^{13}$ Por ello a nivel local se ha observado mediante un estudio de sondeo rápido realizado por las investigadoras, que en la I.E Monseñor Juan Tomis Stack ubicado en el PJ. Túpac Amaru, no se encuentra excluido de la realidad, a nivel macro ambiente es muy resaltante la zona donde se encuentra ubicado, puesto que en sus alrededores existen polvo y tierra. Finalmente se evidenció que las familias que viven alrededor del colegio, persisten en el acumulo de basura, exponiendo al alumnado a contraer algún tipo de enfermedad antes mencionada, también se puede observar en el colegio, los pisos y ventanas con polvo; restos de residuos sólidos.

Frente a esta realidad las investigadoras se plantean el siguiente problema de investigación ¿Cómo influye el programa educativo sobre contaminación por residuos sólidos en el aprendizaje teórico práctico de los estudiantes de sexto grado de la I.E. Monseñor Juan Tomis Stack? 12

Para lo cual se planteó como objetivo general: Determinar la influencia del programa educativo sobre contaminación por residuos sólidos en el aprendizaje teórico práctico de los estudiantes de sexto grado. I.E Monseñor Juan Tomis Stack y como objetivos específicos: Identificar como influye el programa educativo en el aprendizaje teórico sobre contaminación por residuos sólidos en los estudiantes de sexto grado de la IE Monseñor Juan Tomis Stack y Evaluar la influencia del programa educativo sobre ambientales influyen en 85 de las 102 categorías de enfermedades y traumatismos enumeradas en el "Informe sobre la salud en el mundo".7

\section{Metodología}

Esta investigación fue de tipo cuantitativo ${ }^{14}$ en diseño cuasi experimental de pre y post prueba con grupos intactos, ya que se buscó conocer a través de la variable independiente como el programa educativo sobre la contaminación por residuos sólidos, tiene un efecto y relación con la variables dependientes como es la influencia que ejerce en el aprendizaje en los estudiantes del sexto grado; además los sujetos no se asignan al azar a los grupos. Los estudiantes del 6to grado ya estaban asignados en cada sección antes de realizar el programa educativo; donde sí se escogió al azar son las distribución de grupos: 2 secciones ( $A$ y $D$ ) grupo experimental y 3 secciones (B, C, E) grupo control. ${ }^{15}$

Por otro lado, se utilizó una la muestra, no probabilística, es decir que ha sido seleccionada al azar según el criterio de las investigadoras donde existe dos grupos el grupo control que lo constituyeron las secciones B, C y E con 23, 29 y 20 alumnos respectivamente $y$ el grupo experimental (recibirá estímulo) que lo constituyeron las secciones A y D con 22 y 28 alumnos respectivamente. 
El instrumento utilizado fue el cuestionario, constituido por 20 preguntas formuladas en base a las variables independiente y dependiente 18 , evaluadas en forma de pre y post prueba y también la lista de cotejo, que permitió identificar cuidadosamente las mejoras y/o cambios en los hábitos de conservación del medio ambiente por parte de los estudiantes, siendo esto un paso hacia la identificación y realización de progresos.

El instrumento fue probado a través de la prueba piloto con 21 estudiantes del 6to grado B del año anterior, cuyos resultados nos permitió modificar la pregunta 20 , la cual no fue entendida, por otro lado también se realizó la prueba de expertos quienes sugirieron mejorar la redacción de las preguntas $5,6,19,20$ y los datos generales.

En cuando al plan de procesamiento ${ }^{15}$, los instrumentos aplicados a la muestra fueron: el cuestionario y la lista de cotejo que fueron utilizados en un pre y post que se realizó por parte de las investigadoras, una vez que se recolectaron los datos, se procedió a revisar y verificar la estructura de los datos obtenidos, posteriormente se llevó a cabo la edición de estos datos, inmediatamente se procedió a trabajar con el programa de Excel y MegaStat, obteniéndose la creación de tablas, gráficos y/o cuadros para realizar el análisis de datos.

Se aplicó la prueba de hipótesis de diferencia pareada para determinar si el programa educativo ha influenciado en el grupo experimental. Para determinar la prueba de hipótesis de muestras grandes para la diferencia entre dos medias poblacionales 19, cuyo resultado se obtuvo que en un $95 \%$ se confianza se estima que existe diferencia en el nivel de aprendizaje teórico práctico, siendo mayor.

\section{Resultados, análisis y discusión}

\section{Influencia del Programa Educativo sobre Contaminación por residuos sólidos en el Aprendizaje teórico}

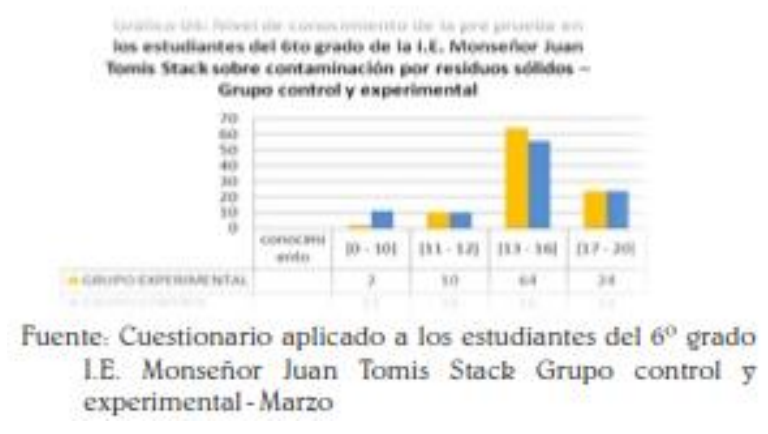

Interpretación: Se observa que el $64 \%$ que corresponde al grupo experimental obtuvieron notas entre 13 - 16, frente al $56 \%$ que obtuvo el grupo control; es decir que ambos grupos presentaron un logro de aprendizaje; sin embargo el grupo experimental obtuvo un porcentaje más elevado debido a la posibilidad de tener un conocimiento más amplio del tema evidenciándose por el $2 \%$ del grupo experimental frente al $11 \%$ del grupo control cuyos resultados fueron las notas de $0-10$, mostrando un inicio de aprendizaje. 


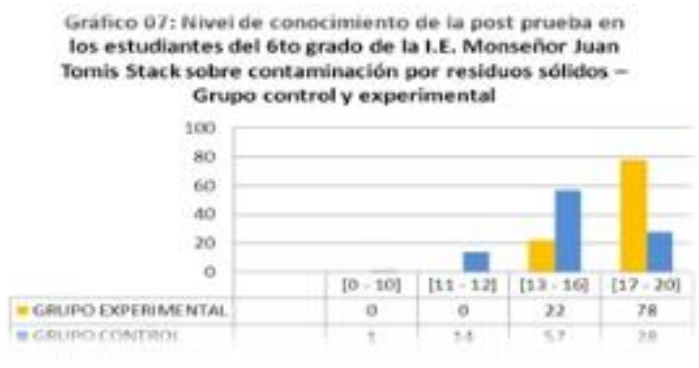

Fuente: Cuestionario aplicado a los estudiantes del $6^{\circ}$ grado I.E. Monseñor Juan Tomis Stack Grupo control y experimental-Julio

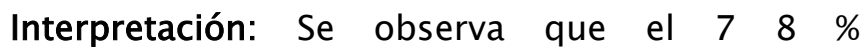
correspondiente al grupo experimental obtuvieron notas entre 17 - 20 logrando un aprendizaje destacado, frente al $28 \%$ que obtuvo al grupo control; es decir que el grupo experimental obtuvo un porcentaje más alto debido a la aplicabilidad del programa educativo que recibió a diferencia del grupo control, además se evidenció un porcentaje 0 a diferencia del $1 \%$ del grupo control cuyas notas fueron entre $0-10$.

\section{Influencia del Programa Educativo sobre contaminación por residuos sólidos en el Aprendizaje práctico.}

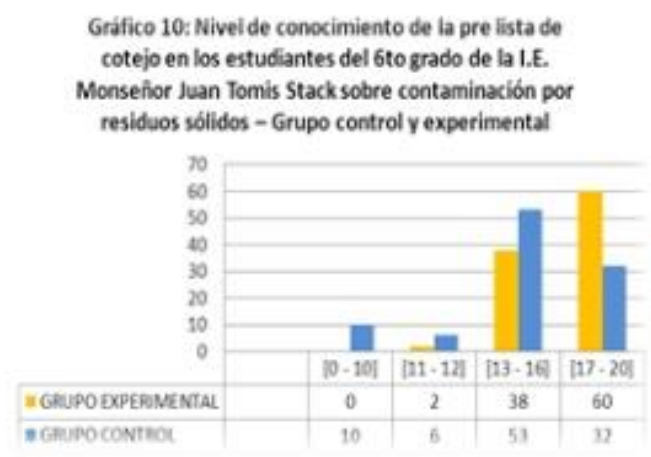

fuente: Lista de cotejo aplicado a los estudiantes del 6 to grado de la 1.E. Monseñor Juan Tomis Stack (Grupo control y experimental) Marzo

Interpretación: Se observa que el $60 \%$ que corresponde al grupo experimental obtuvieron notas entre 17 - 20, frente al 32\% obtenido por el grupo control; es decir que ambos grupos están en proceso de aprendizaje; sin embargo el grupo experimental evidenció un porcentaje más elevado debido a la posibilidad de la aplicación de sus conocimientos sobre el tema.

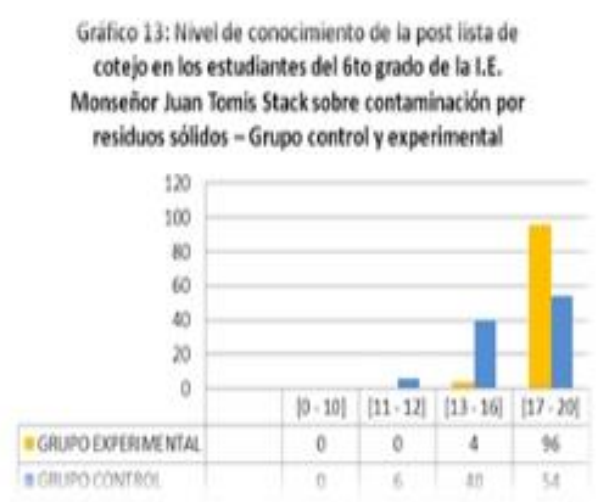

Fuente: Lista de cotejo aplicado a los estudiantes del 6 to grado de la I.E. Monseñor Juan Tomis Stack (grupo control y experimental)-Julio

Interpretación: Se observa que el $96 \%$ que corresponde al grupo experimental obtuvieron notas entre 17 - 20 logrando un aprendizaje destacado, frente al $54 \%$ obtenido por el grupo control; es decir que el grupo experimental obtuvo un porcentaje más alto debido a la aplicación del programa educativo sobre contaminación por residuos sólidos que recibió a diferencia del grupo control, evidenciando a través de los resultados obtenidos en la lista de cotejo además de la observación por parte de las investigadores.

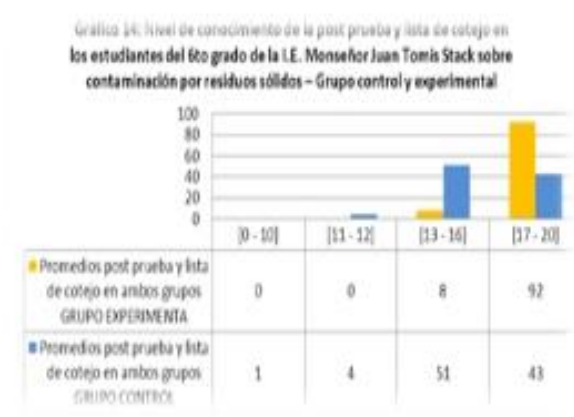

Fuente: Cuestionario y lista de cotejo aplicado a los estudiantes del 6 to grado de la I.E. Monseñor Juan Tomis Stack posterior al programa educativo sobre contaminacion por residuos solidos

Interpretación: Se observa, que el 92\% obtuvieron como promedios notas entre 17 - 20 logrando un aprendizaje teórico práctico destacado, frente al $43 \%$ obtenidos por el grupo control; es decir que el grupo experimental obtuvo un porcentaje más alto debido a la aplicación del programa educativo 
que recibió a diferencia del grupo control, por otro lado ninguno estudiante del grupo experimental. Evidenciando además que la ejecución de programas educativos en conjunto con la estimulación favorece un aprendizaje teórico práctico significativo, y que su continuidad aumenta la posibilidad de adquirir hábitos de conservación ambiental y con ello disminuir tasas de morbi mortalidad a causa de la contaminación por residuos sólidos.

En esta investigación los sujetos de estudio ueron 122 estudiantes del sexto grado de primaria, de las edades entre 10 - 16 años. Según la OMS refiere que el adolescente está comprendido entre las edades de 10 - 19 años, cuyas características no es uniforme y varía de acuerdo a las características individuales y de grupo. ${ }^{21}$ Cabe resaltar que en el Perú la población mayor de 11 años representa el $61,8 \%$ de la población del país. ${ }^{22}$

Por ello es oportuno resaltar que dentro de las características del adolescente este busca tomar conciencia de sí mismo enfrentándose a dificultades en cuanto al manejo del tiempo y del orden $^{23}$. Sin embargo en cuanto al adolescente de hoy (Peruano, Colombiano, etc.) busca preocuparse en construir modas, tener grupos de amigos sin importarles sus hábitos además que es preocupante aptitud por aprender sobre formas de proteger y evitar contaminar el medio ambiente, y por el interés que tiene el adolescente únicamente por el futuro, sin tener en cuenta que a la medida que van tal vez ese futuro no llegue. Asimismo, hacer mención del gran déficit que presenta el estado peruano en cuanto a la implementación de los servicios de educación y salud en este grupo etario ${ }^{22}$. Es evidente decir que las características identificadas en el adolescente de la I. E Juan Tomis Stack, coinciden con las características anteriormente mencionadas.

A partir de lo dicho anteriormente se puede afirmar que el adolescente se encuentra expuesto a factores de riesgo ambientales, resaltando que la vulnerabilidad en esta etapa es mayor, además en este grupo etario la práctica de estilos de vida saludables casi siempre es escasa o nula, lo cual conlleva a altos índices de morbimortalidad ${ }^{24}$ por enfermedades como las diarreicas, pulmonares, de la piel, etc 25 . Desde esta perspectiva el adolescente, debe reconocer que las enfermedades expuestas son a consecuencias de la contaminación ambiental, entendiéndose a este como la presencia de cualquier agente físico, químico o biológico o la combinación de varios agentes nocivos para la salud y dan como resultado el agotamiento de la capa de ozono, el cambio climático y la pérdida de la biodiversidad, empeorando así el crecimiento económico $26,27$.

Así mismo la contaminación medioambiental constituye un grave problema de salud que afecta a los países desarrollados y en desarrollo por igual27, generando diversas enfermedades, que son atribuidos a los riesgos ambientales, y entre la mala calidad del ambiente agua insalubre, saneamiento deficiente y dichas infecciones ${ }^{5,28}$.

En efecto el entorno está relacionado con el impacto en los comportamientos de las personas, resaltando que debe existir la evaluación y control de los factores ambientales que potencialmente pueden afectar la salud; dirigiéndose a la prevención de enfermedades y la creación de ambientes propicios para la salud ${ }^{29}$. En tal sentido cabe resaltar que existen tipos de contaminación ambiental entre ellos la del suelo y del aire, cuyo factor común contaminante son los residuos sólidos $^{30}$, teniendo como producto final la degradación de la calidad natural del medio ambiente 32,33 .

Al haber evidenciado una carencia y/o necesidad, transformándola de esta manera en una motivación para el adolescente, que por deseo propio logre tener un espacio adecuado, limpio y seguro para ellos; se aplicó un programa educativo sobre contaminación por residuos sólidos, pero antes saber que tanto conoce el adolescente sobre el tema mediante un cuestionario dando como resultados reflejados en el gráfico 04 donde el $64 \%$ correspondiente al grupo experimental (aulas A y 
D) obteniendo notas entre 13 - 16 y frente al $56 \%$ obtenidos por el grupo control (aulas B, C y E).

Comparando los resultados obtenidos tanto en el grupo experimental como en el control se observó que a pesar que todo el 6 to grado reciben la misma educación en la asignatura correspondiente, existe un porcentaje de desaprobados que se evidencia por un $2 \%$ en el grupo experimental y el $11 \%$ en el grupo control. Se aplicó el programa educativo sobre contaminación por residuos sólidos, al finalizar se aplicó nuevamente el cuestionario dando como resultado en el gráfico 07 que del $100 \%$ de los estudiantes, el $78 \%$ correspondiente al grupo experimental obtuvieron notas entre $17-20$ logrando un aprendizaje destacado, frente al $28 \%$ que obtuvo al grupo control; es decir que el grupo experimental obtuvo un porcentaje más alto debido a la aplicabilidad del programa educativo sobre contaminación por residuos sólidos (estímulo) que recibió a diferencia del grupo control. Esto se confirma con la prueba de hipótesis de diferencia pareadas donde con un margen de error de $0.05 \%$ se rechaza la hipótesis nula, y se acepta la hipótesis alternativa al 95\% de confianza y se estima que el programa educativo en contaminación por residuos sólidos tuvo un efecto significativo en el aprendizaje teórico de los estudiantes del sexto grado.

Es justo decir que ligado al conocimiento, es muy importante saber si los estudiantes aplican lo que saben, si el estímulo es constante en ellos por parte de sus profesores, es por ello que también se vio la necesidad de aplicar una lista de cotejo antes de ejecutar el programa educativo cuyos resultados se evidencia en el gráfico 10 donde el $60 \%$ correspondiente al grupo experimental obtuvieron notas entre 17-20, frente al $32 \%$ del grupo control notas entre $17-20$. En este contexto de contrariedad se llegó a la conclusión de que las diferencias de desaprobados, se debió a que el grupo control conformado por el aula E estuvo constituido por algunos alumnos repitentes, además de presentar algunos problemas de conducta y variedad en cantidad de inasistencias a comparación de las otras secciones.

Teniendo una visión diagnóstica en cuanto a la práctica sobre lo que se aprendió del programa educativo se aplicó nuevamente la lista de cotejo cuyos resultados se reflejan en el gráfico 13 donde el $96 \%$ que corresponde al grupo experimental obtuvieron notas entre $17-20$ logrando un aprendizaje destacado, frente al $54 \%$ obtenido por el grupo control; es decir que mientras el conocimiento sea una vía fluida y clara, su aplicabilidad en nuestras vida formará un hábito positivo y con ello lograr el cambio para mantener nuestra vida y ambiente saludable

Esto se confirma con la prueba de hipótesis de diferencias pareadas donde con un margen de error del $0.05 \%$ por lo que se rechaza la hipótesis nula y acepta la hipótesis alternativa al 95\% de confianza estimándose que la aplicabilidad del programa sobre contaminación por residuos sólidos tuvo un efecto significativo en el aprendizaje práctico de los estudiantes del sexto grado.

Finalmente se calculó los promedios tanto de la post prueba como de la lista de cotejo (grupo control y experimental), cuyos resultados se reflejan en el gráfico 14 donde el $92 \%$ obtuvieron como promedios notas entre 17 - 20 logrando un aprendizaje teórico práctico destacado, frente al $43 \%$ obtenidos por el grupo control; es decir que el grupo experimental obtuvo un porcentaje más alto debido a la aplicación del programa educativo sobre contaminación por residuos sólidos que recibió a diferencia del grupo control.

Evidenciando además que la ejecución de programas educativos en conjunto con la estimulación favorece un aprendizaje teórico práctico significativo, y que su continuidad aumenta la posibilidad de adquirir hábitos de conservación ambiental y con ello disminuir tasas de morbi mortalidad a causa de la contaminación por residuos sólidos. 
Así mismo se aplicó la prueba de hipótesis de muestras grandes para la diferencia entre dos medias poblacionales, para determinar la diferencia en cuanto al nivel de aprendizaje teórico- práctico después de aplicar el programa educativo entre el grupo experimental quien recibió el estímulo y el grupo control, donde con margen de error de $0.05 \%$ se rechaza la hipótesis nula, y acepta la hipótesis alternativa llegando a la conclusión que en un $95 \%$ de confianza se estima que existe diferencia en el nivel de aprendizaje teórico práctico, siendo mayor en el grupo experimental que en el grupo control.

Estos resultados se comparan y se confirma con lainvestigaciones realizado en México, titulado Manejo de residuos sólidos en instituciones educativas, cuyos resultados fueron que por medio de sesiones de clases se motivaron al alumno a colocar la basura en los contenedores implementados 37; es así que en la presente investigación se evidenció los mismos resultados ya que los estudiantes mediantes las sesiones dadas como parte del programa educativo, permitió la reducción de papeles, cáscaras de frutas y restos de lápices tajados en el aula, para colocarlos en los distintos contenedores de basura

(3) según la característica de los residuos sólidos esto se confirma y se apoya en que el conocimiento y la motivación hacen de la prácticas un hábito positivo.

Así mismo en Colombia en la investigación Educación Ambiental para la Participación en la Gestión de Residuos Sólidos, sus resultados mejoraron los espacios comunes y redujeron notablemente la basura 38. Por ello se afirma y complementa con lo dicho que utilizando adecuadamente los materiales de papel u otros, se reduce de esta manera la presencia de residuos dispersos en el aula, evidenciando una mejor amplitud, conformidad, en pocas palabras en un ambiente ordenado, limpio y saludable Por otro lado resaltar que el aprendizaje tanto teórico como práctico del adolescente está muy ligado a su capacidad cognitiva y a la actitud que este tiene frente a su persona y el entorno, por ello se tiene en claro que el aprendizaje en inicio empieza por sus dudas, luego cuestiona ideas u opiniones de sus padres; pero son conscientes de que esas opiniones no son suyas e intentan buscar sus propias verdades, las cuales surgirán de su propio desarrollo intelectual34. Pues bien para tratar dicho desarrollo y lograr un aprendizaje utiliza su cerebro (desarrolla hasta alcanzar los 20 años de edad), cuya materia gris incrementa su volumen durante las edades más tempranas pero durante la pubertad esta comienza a disminuir y adelgazarse. Logrando así una organización más eficiente del cerebro, aumentando así la capacidad de las neuronas para transmitir el impulsos ${ }^{35}$.

Considerando la constitución anatomo-fisiológica del adolescente, se observa las habilidades cognitivas como la atención y madurez cerebral, logrando así un mejor aprendizaje teóricopráctico, comprendiendo con mayor claridad su entorno, analizando las causas -consecuencias y aplicando estilos de vida saludables que ayuden a complementar un aprendizaje significativo.

En este mismo sentido los estilos de vida deben ser siempre saludables y el hábito de conservar el medio ambiente deber ser una prioridad, asumiendo la sensibilidad que estos tienen por captar lo que pasa en su entorno a través de sus sentidos son muy amplios.

Por ello se puede asegurar que el programa educativo tuvo un efecto significativo incrementando el nivel de aprendizaje teórico práctico, en los estudiantes del grupo experimental obteniendo un logro destacado (1720). Y en cuanto al grupo control, en algunos casos han variado ligeramente el resultado de la post prueba y post lista de cotejo, debido a que este grupo no recibió estímulo (programa educativo), convirtiéndola un grupo con mayor vulnerabilidad a los factores de riesgos (contaminación por residuos sólidos), resaltando que el cambio parte desde poblaciones pequeñas; y con apoyo de las instituciones públicas. 


\section{Conclusiones}

El programa educativo sobre contaminación por residuos sólidos tuvo un efecto significativo demostrada con la prueba de hipótesis de diferencia pareadas al $95 \%$ de confianza, evidenciándose en el incremento del nivel de aprendizaje teórico práctico en los estudiantes del grupo experimental, obteniéndose un logro destacado del $92 \%$.

El programa educativo sobre contaminación por residuos sólidos tuvo un efecto significativo, en el aprendizaje del estudiante, observándose que antes de aplicar el programa educativo el $24 \%$ de estudiantes obtuvieron un logro destacado, después de aplicado el programa aumentó 54\%, evidenciándose un logro destacado en su $78 \%$.

El programa educativo sobre contaminación por residuos sólidos tuvo un efecto significativo en el aprendizaje practico de los estudiantes, cuyo porcentaje antes de aplicar el programa educativo fue del $60 \%$ obtuvieron un logro destacado posteriormente se observó un aumento del 36\%, dando un logro destacado en su $96 \%$.

Lo que permite afirmar que el cerebro del estudiante está predispuesto a recibir información y a grabarla para ser aplicado, en ello juega un papel importante el profesor, recordar su aplicación práctica en la conservación del medio ambiente influyendo de esta manera en su formación y en la protección de su salud

\section{Bibliografía}

1. Elpais.com. La destrucción del medio ambiente no tiene precedentes, según WWF. [sede Web]. Madrid: Prisa; 2006 - [acceso 05 de noviembre del 2011]. Disponible en:

http://www.elpais.com/articulo/sociedad/destruc cion/medio/ambiente/tiene/precedentes/WWF/el pepisoc/20061025elpepisoc_8/Tes
2. Sistema de las Naciones Unidad en el Perú. [sede Web]*. Perú: UNEP; 2010 [acceso 05 de noviembre del 2011]. Medio ambiente y cambio climático. Disponible en:

http://www.onu.org.pe/Publico/infocus/medioam biente.aspx

3. Zabalegui A. El rol del profesional de enfermería. Aquichan [revista en Internet]* 2003 Agosto Diciembre. [acceso 05 de noviembre del 2011]; 3(1). Disponible en:

http:/ / redalyc.uaemex.mx/src/inicio/ArtPdfRed.js p?iCve $=74130304$

4. Scribd.com. Política de asentamientos humanos en áreas protegidas de peten. Guatemala: scribd.com; 2002 - [acceso 29 de agosto del 2011 ]. Disponible en:

http://es.scribd.com/doc/48473261/politicaasentamientos-humanos-final

5. Chelala C. Impacto del ambiente sobre la salud infantil. Organización panamericana de la salud [revista en Internet]* 1999 Agosto - Diciembre. [acceso 04 de septiembre del 2011]; 36(3). Disponible en:

http://www.paho.org/spanish/hep/infancia. Pdf

6. Educación y gestión ambiental. [sede Web]*. Chile: Sociedad misionera de san Columbano; 2008. [acceso 10 de septiembre del 2011]. Programa de educación ambiental, manejo de residuos sólidos escolares, colegio polivalente Elisa Valdez. Disponible en :

http:/ / compostcev.blogspot.com/

7. Organización Mundial de la Salud. Ambientes saludables y prevención de enfermedades. Informe de un Grupo Científico de la OMS. Ginebra: OMS; 2006. Serie de Informes Técnicos: 978.

8. Ministerio del ambiente. Informe de la situación actual de la gestión de residuos sólidos municipales. El Perú avanza; 2008. 
9. Aspectos del medio ambiente. En: Proyecto villa verde. Gerencia de servicios ambientales de la municipalidad de villa el salvador. Perú: Villa el Salvador; 2003. P.D1 - D45.

10. Estado situacional del medio ambiente. [Sede Web]*. [Acceso 25 de octubre de 2011 ]. Estado situacional del medio ambiente en la región Lambayeque. Disponible en:

http://www.mesadeconcertacion.org.pe/do cumentos/regional/Lambayeque/r14_0115 $0 . p d f$

11. Cárdenas R. Aumento de enfermedades por contaminación. 2010. [acceso 13 de septiembre del 2011 ]. Disponible en:

http://impactobio.blogspot.com/2010/11/au mento-de-enfermedades-por.html

12. Blogspot. com. Contaminación ambiental: Blogspot.com; 2006 - [actualizada el 2009; acceso 04 de septiembre del 2011]. Disponible en: http: //contaminacion-ambiente.blogspot.com/

13. Zevallos H., Aceituno P. Manejo de residuos sólidos. $2^{\circ}$ Ed. Perú: ADRA PERU; 2008

14. Bernal C. Metodología de la investigación: para administración, económica, humanidades y ciencias sociales. 2 edicion. México: Pearson educación; 2006

15. Hernández R., Fernández C., Baptista P. Metodología de la Investigación. Ed. 2da. México: editorial McGraw.Hill Interamericana S.A. de C.V; 1998.

16. Armitage E, Berry G. Estadística para la investigación biomédica. España: Elsevier; 1997

17. Barahona A, Barahona F. Metodología del trabajo científico. 4ta Ed. Colombia: editorial IPLER Ltda;1984
18. Martinez B, Cespedes N. Metodologis de la investigacion. Estrategias para investigar. 1. ${ }^{\circ}$ ed. Lima. Editorial Sanchez, 2008.

19. Scribd.com. Prueba de hipótesis para muestra pareada. 2002 - [acceso 15 de septiembre del 2011 ]. Disponible en:

http:/ /es.scribd.com/doc/6784208/Prueba-deHipotesis-Para-Muestras-Pareadas

20. Catholic.net. Bioética personalista. [sede Web]. 2011 - [acceso 08 de noviembre del 2011 1]. Disponible en:

http://es.catholic.net/sexualidadybioetica/371/2 508/articulo. php?id $=4813$

21 Organización Mundial de la Salud. [Sede Web]*. Acceso 3 de septiembre de 2012] Salud de los adolescentes. Disponible en:

http://www.who.int/topics/adolescent_health/es/

22. Colegio de Psicólogos del Perú. [revista en Internet]*2010 enero -diciembre. [Acceso 10 de septiembre del 2012]; 9(1). Disponible en: http://revistas.concytec.gob.pe/pdf/rppsi/v9n 1/ a05v9n1.pdf

23. Gómez P. Niño sano. 3 edicion. Colombia: Editorial medica panamericana; 2005

24. Giraldo A., León C, Navarro Y, Trejos O. Determinación de prácticas de autocuidado $y$ prácticas de riesgo en los patrones nutricionales y de eliminación en un grupo de estudiantes del programa de enfermería de la universidad de Caldas. [revista en Internet]*1997 agosto noviembre. [Acceso 10 de abril del 2012]; 3(3).

Disponible en: http://promocionsalud.ucaldas.edu.co/download s/Revista\%207_13.pdf

25. Solís L, López J. Principios básicos de contaminación ambiental. México: Editorial Instituto literario; 2003.

26. La contaminación ambiental. [Sede Web]*. 
[Acceso 10 de Octubre de 2011]. Contaminación ambiental nacional. Disponible en:

http://www.peruecologico.com.pe/lib_c22_t04.ht $\mathrm{m}$

27. Antenasanluis.com. [Sede Web]* . [Acceso 4 de septiembre de 2012]Calidad del aire y Salud.

Disponible en:

http://www.antenasanluis.com/noticia/nota/ 176

7-calidad-del-aire-y-salud.html

28. Zonacatastrófica.com. Enfermedades consecuencia de la contaminación ambiental [sede Web]. Red blogia; 2008 - [acceso 05 de noviembre del 2011]. Disponible en:

http://www.zonacatastrofica.com/nuevas-

consecuencias-de-la-contaminacion.html

29. Organización Mundial de la Salud. [Sede Web]*. [Acceso 5 de septiembre de 2012]Salud ambiental. Disponible en:

http://www.who.int/topics/environmental_health /es/

30. De igual a igual [sede Web]*. Ecoportal; 2007 [actualizada 09 de Febrero de 2010; acceso 05 de noviembre del 2011]. Problemas de la contaminación ambiental. Disponible en: http://www.deigualaigual.net/derechoshumanos/61-medio-ambiente/1359-

contaminacion-ambiental

31. Fondo Nacional de Ambiente. [Sede Web]*. Perú: FONAM; 2010 [acceso 27 de Septiembre de 2011 ]. Residuos sólidos. Disponible en: http://www.fonamperu.org/general/agua/residuo s.php

32. Fraume N. Diccionario ambiental. Editorial ECOE: Colombia, 2007

33. Tapia L, Quintana E, Morales C. Calidad de Vida en Potosí: Efectos ambientales. Bolivia: Editorial Wilmer Urrelo; 2002

34. Cepsi.com. [Sede Web]". [Acceso 3 de septiembre de 2012] Desarrollo cognitivo: el pensamiento del adolescente. Disponible en:

http://www.cepvi.com/articulos/adolescencia2.sh tml

35. SNF. [Sede Web]*. [Acceso 4 de septiembre de 2012] El cerebro adolescente: haciendo una

diferencia hoy. Disponible en: http://www.sfn.org/siteobjects/published/0000B DF20016F63800FD712C30FA42DD/2CE21 46A61 8B9870E2E76E5454733378/file/EI_cerebro_adole scente.pdf

36. Estilos de aprender. [Sede Web]*. [Acceso 5 de septiembre de 2012]Bases de aprendizaje.

Disponible en:

http:/ / www.slideshare.net/augustoeliasc/descrip cion-de-los-estilos-de-aprender-presentation

37. Cruz B, Teutli L, González A, Jiménez G. Manejo de residuos sólidos en instituciones educativas. [Internet]. Puebla: Universidad Autónoma de Puebla. [actualizada en 2005; acceso 15 de septiembre del 2011]. Disponible en:

http://www.uaemex.mx/Red_Ambientales/docs/ memorias/Extenso/PA/EC/PAC-03.pdf

38. Pelayo L, García R, Pérez C. Educación Ambiental para la Participación en la Gestión de Residuos Sólidos en un Centro Universitario [Internet]. Colombia: Universidad de Guadalajara. [actualizado en 2011; acceso 30 de abril del 2012]. Disponible en:

http:/ / www.laccei.org/LACCEI2011-

Medellin/RefereedPapers/EUEE239_Pelayo.pdf 